\title{
Drug Resistance Pattern of Bacterial Pathogens of Enterobacteriaceae Family
}

\author{
Bhola Shankar Sah $^{1 *}$, Manita Aryal ${ }^{1}$, Dipak Bhargava ${ }^{2}$, Amrullah Siddique $^{2}$ \\ ${ }^{1}$ Central Department of Microbiology, Tribhuvan University, Kirtipur, Nepal and \\ ${ }^{2}$ National Medical College and Teaching Hospital, Birgunj, Nepal
}

*Corresponding author: Bhola Shankar Sah, Central Department of Microbiology, Tribhuvan University, Kirtipur, Nepal; Email: bholashankarsah999@gmail.com

\begin{abstract}
Objectives: This study was done to determine the drug resistance pattern and Extended Spectrum $\beta$-Lactamase (ESBL) in bacterial isolates of Enterobacteriaceae family from different clinical specimens.

Methods: The isolates were identified by conventional culture techniques and subjected to antimicrobial susceptibility testing by modified Kirby Bauer disk diffusion methods and ESBL detection by combined disk method.

Results: Of the total 1602 sample processed 200 (12.5\%) bacteria of Enterobacteriaceae family were isolated and $85.5 \%$ of them were multidrug resistant. Of the total Enterobacteriaceae isolates $27 \%$ were ESBL producers. Single isolate of stool was MDR and ESBL producer. Higher prevalence of MDR isolates (100\%) and ESBL producer (41.2\%) was observed in sputum specimen. Higher multidrug resistance (92.1\%) and ESBL production (35\%) was detected in Klebsiella pneumoniae.

Conclusion: The most effective antibiotics towards the isolates of Enterobacteriaceae were imipenem, amikacin, chloramphenicol and tetracycline. Emergence of MDR and ESBL producing Enterobacteriaceae requires proper infection control measures and routine and reliable detection of ESBL with rationale use of antibiotics.
\end{abstract}

Key words: Enterobacteriaceae, Multidrug resistance, ESBL, Antibiotic susceptibility testing.

\section{INTRODUCTION}

The prevalence of multidrug resistance (MDR) bacterial species has increased considerably since the introduction followed by arbitrary use of new generation extended spectrum antibiotics like third and fourth generation cephalosporins, carbapenems, monobactams, broad and extended spectrum penicillins and other antibiotics (Fang et al. 2008).

Multidrug resistance bacterial isolates have been frequently reported from different parts of the world as an emergence of treatment problem. The World Health Organization (WHO), the European Commission (EU), and the U.S. Centers for Disease Control and Prevention (CDC) have recognized the importance of studying the emergence and determinants of multidrug resistance as well as the need for control (Alekshum 2007). Citrobacter, Enterobacter, Escherichia, Klebsiella, Proteus, Salmonella, Serratia, Shigella, Yersinia, etc. are the medically important genera of the family
Enterobacteriaceae (Gray 1995).

Extended spectrum $\beta$-lactamases (ESBLs) in Enterobacteriaceae is defined as $\beta$-lactamases capable of hydrolyzing penicillins, broad and extendedspectrum cephalosporins and monobactams and are inhibited by clavulanic acid. They are generally derived from TEM and SHV-type enzymes. ESBLs are often located on plasmids that are transferable from strain to strain and between bacterial species. ESBLsproducing Enterobacteriaceae have been responsible for numerous outbreaks of infection throughout the world and pose challenging infection control issues (Rupp and Fey 2003).

Antibiotic resistance is one of the alarming issues, affecting human health. There are various factors responsible to the emergence of resistance such as misuse and overuse of antibiotics, patients related factors, inappropriate prescriptions by physicians, self-medications especially young adults, use of broad 
spectrum antibiotics and synergistic combinations, unnecessary promotion by pharmaceutical industry, and lack of awareness with the new guidelines recommended for antimicrobial testing, etc. Multidrug resistance is getting common phenomenon and are being reported worldwide (Khan et al. 2014).

Multidrug Resistance among bacteria is one of the greatest challenges in the field of medicine. Resistance mechanism to different classes of antibiotics such as tetracyclines, aminoglycosides and cotrimoxazole is of bigger issue. However, broad spectrum resistance to $\beta$-lactam and fluoroquinolones are of utmost significance. In the early 1950s, enteric bacteria that mediated resistance to first penicillin attracted attention of the researchers. The introduction of $3^{\text {rd }}$ generation cephalosporins was milestone in antimicrobial chemotherapy but after few years resistance to these drugs was observed in different species (Sanders and Sanders 1987).

The outbreaks of infectious disease caused by known commensal and pathogenic bacteria by acquisition of new resistance determinants have eluded the action of multiple antibiotics. The development of resistance in the responsible pathogens has worsened the situation and very little resources to investigate and provide reliable data. The emergence of multiple drug resistance in gastrointestinal tract infection and several other infections has had its greatest toll in developing countries. This condition has large population around the globe at great risk of numerous infections and even greatest risk of acquiring nosocomial infection caused by MDR isolates of Gram negative bacteria (Walsh 2003; Byarugaba 2004).

Extended spectrum $\beta$-lactamase (ESBL)-producing Enterobacteriaceae pose unique challenges to clinical microbiologists, clinicians, infection control professionals and antibacterial-discovery scientists. Incidence of multidrug resistant and extended spectrum $\beta$-lactamases producing Enterobacteriaceae has been rising in several parts of the world and has been associated with high morbidity and mortality. Through this research an effort was made to find out the prevalence of bacterial pathogens of Enterobacteriaceae family in different clinical specimens. This research also conducted the antimicrobial susceptibility test of isolated Enterobacteriaceae and assessed the burden of multidrug resistant and extended spectrum $\beta$-lactamases producers among the isolates. Bacterial pathogens of Enterobacteriaceae family have received much more attention in recent years because of their involvement in number of severe infections and multiple drug resistance patterns. Much of the study regarding such bacterial infections is limited to Kathmandu valley. So an effort was made to assess the burden of infection caused by Enterobacteriaceae and their antibiotic susceptibility pattern around Birgunj city. It is aimed that this study will aid in treatment procedures to patient suffering from infections of Enterobacteriaceae from that region.

\section{MATERIALS AND METHODS}

Study population: A prospective cross-sectional study was performed for 6 months (14/10/2015 to 13/04/2016) at National Medical College and Teaching Hospital, Birgunj. This study included patients of all age group and both sexes visiting this hospital from whom samples were sent for routine culture and antibiotic susceptibility testing.

Conventional microbiological tests: The identification of various gram negative isolates was done using standard microbiological techniques which comprises of studying of colonial morphology, staining reactions and various biochemical properties. Isolated colonies from the pure culture were identified by standard conventional biochemical tests. Antibiotic susceptibility testing was performed by using Kirby-Bauer disk diffusion method on Mueller-Hinton agar. Screening tests for ESBL detection was done according to the CLSI guidelines and confirmed by combination disk method.

\section{RESULTS}

A total of 1602 clinical specimen were investigated during a six-month study period, of which 877 (54.7\%) and $725(45.3 \%)$ were from female and male patients respectively. Among 1602 specimens, 1192 (74.4\%) were from indoor patients and $410(25.6 \%)$ specimens from outdoor patients were included in the study.

\section{Distribution of total clinical specimens under investigation}

Among the total 1602 clinical specimens analyzed, 649 (40.5\%) were urine, 360 (22.5\%) were pus, 329 (20.5\%) were blood, $169(10.5 \%)$ were sputum, 84 (5.2\%) were body fluids, $6(0.4 \%)$ were stool and $5(0.3 \%)$ were ET tip and secretion specimens.

A total of $403(25.15 \%)$ bacteria was isolated from 
1602 different clinical specimens among which 255 (63.3\%) isolates were Gram negative while 148 (36.7\%) were Gram positive bacteria. Among the total isolates of Gram negative 200 (75.5\%) belong to the family Enterobacteriaceae, 45 (19.6\%) were P. aeruginosa and 10 (3.9\%) were Acinetobacter spp.

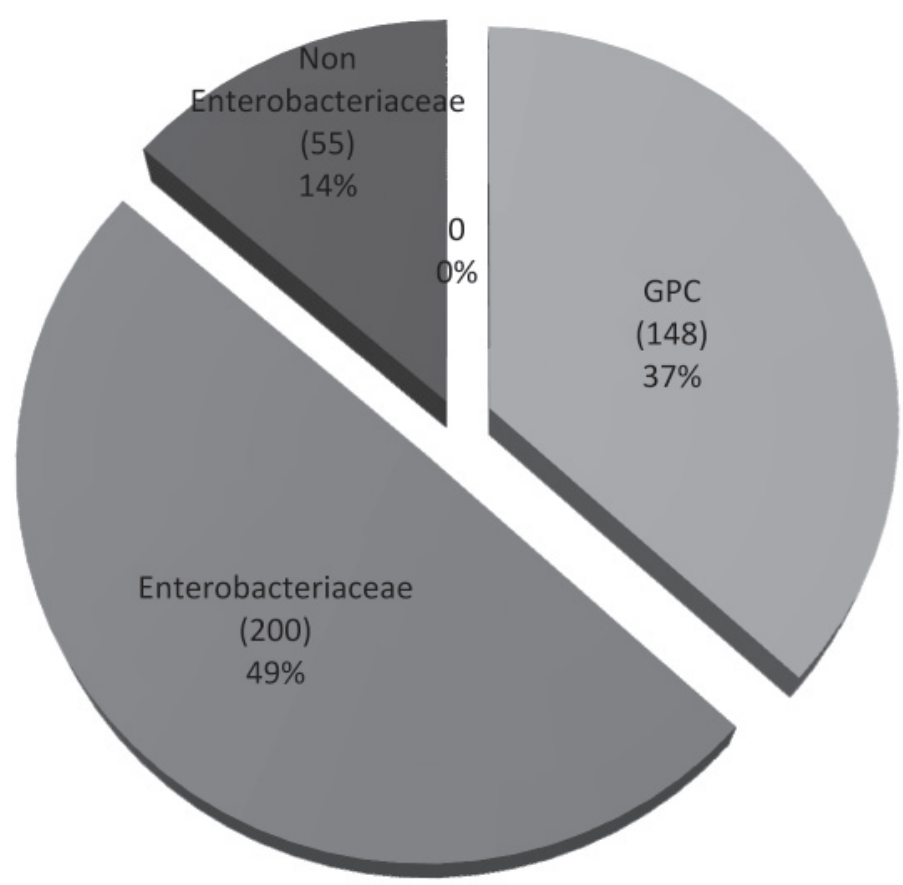

Figure 1: Prevalence of different types of bacteria

Among the total 200 isolates of Enterobacteriaceae E. coli was the most predominant isolate with $56.5 \%$ followed by K. pneumoniae (31.5\%), P. mirabilis (5.5\%), P. vulgaris
(3.5\%), Enterobacter spp. (1.5\%), 0.5\% Citrobacter spp., $0.5 \%$ S. Paratyphi A and $0.5 \%$ Serratia spp. (Figure 1).

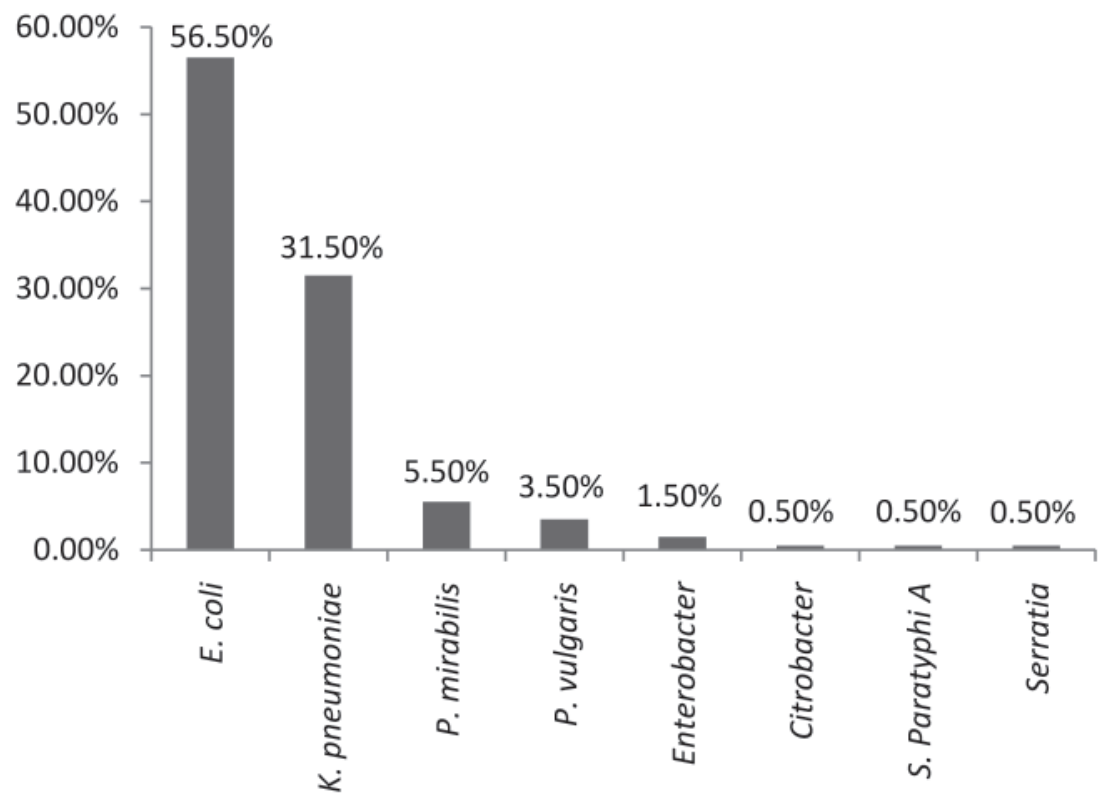

Figure 2: Members of Isolated Enterobacteriaceae 
Antibiotic susceptibility pattern of members of Enterobacteriaceae from various specimens

All the isolated strains of Enterobacteriaceae from different clinical specimens were tested with specific antibiotics by using Kirby - Bauer disk diffusion method. According to CLSI 2014, a set of 12 antibiotics were used to determine antibiotic susceptibility pattern of members of Enterobacteriaceae family.

Imipenem was found most effective antibiotic against members of Enterobacteriaceae with sensitivity $89.5 \%$
(179/200). They showed $73 \%(146 / 200)$ sensitivity towards amikacin, followed by chloramphenicol $69.5 \%(139 / 200)$ and tetracycline $52.5 \%$ (105/200). Nitrofurantoin was found to be effective among $77.9 \%$ (88/113) of the urinary isolates. Ampicillin with $98.5 \%$ (197/200) resistance was the least effective drugs towards the isolates of Enterobacteriaceae followed by cefixime 80.5\%(161/200), amoxyclav 77\% (154/200), ceftriaxone $73.5 \%$ (147/200), cefotaxime $72 \%$ (144/200), cefoxitin $64.5 \%(129 / 200)$ and ciprofloxacin and cotrimoxazole with $58 \%(116 / 200)$ resistance each.

Table 5: Antibiotic susceptibility pattern of Enterobacteriaceae from various specimen

\begin{tabular}{llccccccc}
\hline \multirow{2}{*}{ S.N } & \multirow{2}{*}{ Antibiotic } & \multicolumn{2}{c}{ Sensitive } & \multicolumn{2}{c}{ Intermediate } & \multicolumn{2}{c}{ Resistant } & \multirow{2}{*}{ Total } \\
& & N & $\%$ & $\mathbf{N}$ & $\%$ & N & $\%$ & \\
\hline 1. & Imipenem & 179 & $89.5 \%$ & 7 & $3.5 \%$ & 14 & $7 \%$ & 200 \\
2. & Amikacin & 146 & $73 \%$ & 5 & $2.5 \%$ & 49 & $24.5 \%$ & 200 \\
3. & Chloramphenicol & 139 & $69.5 \%$ & 15 & $7.5 \%$ & 46 & $23 \%$ & 200 \\
4. & Tetracycline & 105 & $52.5 \%$ & 26 & $13 \%$ & 69 & $34.5 \%$ & 200 \\
5. & Nitrofurantoin & 88 & $77.9 \%$ & 13 & $11.5 \%$ & 12 & $10.6 \%$ & 113 \\
6. & Ciprofloxacin & 74 & $37 \%$ & 10 & $5 \%$ & 116 & $58 \%$ & 200 \\
7. & Cotrimoxazole & 73 & $36.5 \%$ & 11 & $5.5 \%$ & 116 & $58 \%$ & 200 \\
8. & Cefoxitin & 58 & $28 \%$ & 12 & $6 \%$ & 130 & $65 \%$ & 200 \\
9. & Cefotaxime & 56 & $28 \%$ & 0 & $0 \%$ & 144 & $72 \%$ & 200 \\
10. & Ceftriaxone & 40 & $20 \%$ & 13 & $6.5 \%$ & 147 & $73.5 \%$ & 200 \\
11. & Cefixime & 33 & $16.5 \%$ & 6 & $3 \%$ & 161 & $80.5 \%$ & 200 \\
12. & Amoxyclav & 26 & $13 \%$ & 19 & $9.5 \%$ & 155 & $77.5 \%$ & 200 \\
13. & Ampicillin & 3 & $1.5 \%$ & 0 & $0 \%$ & 197 & $98.5 \%$ & 200 \\
\hline
\end{tabular}

MDR Enterobacteriaceae isolates in different clinical specimens

Out of total 200 isolates, $171(85.5 \%)$ isolates were multidrug resistant among which all the isolates from sputum and stool specimens showed $100 \%$ multidrug resistance while blood specimens showed 93.3\% MDR followed by urine $(84.1 \%)$, and pus $(81.5 \%)$.

Table 6: MDR Enterobacteriaceae isolates in different clinical specimens

\begin{tabular}{lcccc}
\hline & & MDR & NON-MDR & TOTAL \\
\hline \multirow{4}{*}{ Sample } & Urine & $95(84.1 \%)$ & $18(15.9 \%)$ & 113 \\
& Pus & $44(81.5 \%)$ & $10(18.5 \%)$ & 54 \\
& Blood & $14(93.3 \%)$ & $1(6.7 \%)$ & 15 \\
& Sputum & $17(100 \%)$ & $0(0 \%)$ & 17 \\
\hline TOTAL & Stool & $1(100 \%)$ & $0(0 \%)$ & 1 \\
\hline
\end{tabular}

ESBL production profile of Enterobacteriaceae in different clinical specimens

Out of 200 isolates of Enterobacteriaceae, 54 (27\%) isolates were ESBL producer. Single isolate of stool $(S$.
Paratyphi A) was an ESBL producer. High prevalence of ESBL producer was observed in sputum with $41.2 \%$ followed by pus (31.5\%), blood (26.7\%) and urine (22\%). 
Table 7: ESBL production profile of Enterobacteriaceae in different clinical specimens

\begin{tabular}{lccc}
\hline \multirow{2}{*}{ Samples } & \multicolumn{2}{c}{ No of ESBL } & \multirow{2}{*}{ Total } \\
\cline { 2 - 3 } & Positive (\%) & Negative (\%) & 113 \\
\hline Urine & $25(22.1)$ & $88(77.9)$ & 54 \\
Pus & $17(31.5)$ & $37(88.5)$ & 15 \\
Blood & $4(26.7)$ & $11(73.3)$ & 17 \\
Sputum & $7(41.2)$ & $10(58.8)$ & 1 \\
Stool & $1(100)$ & $0(0)$ & 200 \\
\hline Total & $\mathbf{5 4 ( 2 7 )}$ & $\mathbf{1 4 6 ( 7 3 )}$ & \\
\hline
\end{tabular}

Multidrug resistance and ESBL production profile of Enterobacteriaceae

MDR Enterobacteriaceae were identified by their antibiotic sensitivity pattern. Out of the total 200 Enterobacteriaceae $171(85.5 \%)$ isolates were MDR. E. coli was the most predominant among the total MDR isolates. Single isolates of Citrobacter spp. and $S$. Paratyphi A were MDR strain. The highest percentage of MDR strains among each bacterial isolates were $K$. pneumoniae with $92.1 \%$ multidrug resistance followed by P. vulgaris (85.7\%), E. coli (83.2\%), P. mirabilis (81.9\%) and Enterobacter spp. (66.7\%). The MDR isolates of Enterobacteriaceae was suspected as ESBL producer on the basis of reduced susceptibility to at least one screening agent i.e, cefotaxime or ceftriaxone. Of the total Enterobacteriaceae (200) isolates, 147 isolates were suscpected of being ESBL producer. Of the total 147 screen positive isolates for ESBL production, 54 $(36.7 \%)$ isolates were found to be ESBL producer. The prevalence of ESBL producer among total isolates was $27 \%(54 / 200)$ whereas the prevalence of ESBL producers among MDR isolates was 27.5\% (47/171) and among non-MDR isolates was $24.1 \%(7 / 29)$. Among the total ESBL positive isolates E. coli was the most predominant isolate with 51.9\% (28/54). Among each isolates higher prevalence of ESBL producer was detected in K. pneumoniae with 35\% (22/63) followed by P. mirabilis $27 \%(3 / 11)$ and E. coli $24 . \%(2 / 113)$. Single isolate S. Paratyphi A $100 \%$ were ESBL producer (1/1). All isolates of P. vulgaris, Enterobacter spp., Citrobacter spp. and Serratia spp. were ESBL non-producer (Table 7).

Table 8: Multidrug resistance and ESBL production profile of Enterobacteriaceae

\begin{tabular}{lccc}
\hline & & ESBL & \\
\cline { 2 - 4 } & Positive & Negative & Total \\
\hline MDR & 47 & 124 & 171 \\
NON-MDR & 7 & 22 & 29 \\
\hline TOTAL & 54 & 146 & 200 \\
\hline
\end{tabular}

\section{DISCUSSION}

Bacterial pathogens of Enterobacteriaceae family are often associated with diffferent human infections like respiratory tract infections, blood stream infections, meningitis, endocarditis, urinary tract infections, skin, soft tissue and bone infections, etc.The emergence of ESBLs producing bacteria, particularly K. pneumoniae and E. coli, is now a critical concern for the development of therapies against bacterial infection (Canton and Coque 2006).

A total of 1602 different clinical specimens from the patients of OPD, various wards (medical, surgical, paediatric, emergency observations and ICUs of the hospital that were sent for the microbiological investigation were analyzed. Urine $(40.5 \%)$ was the most frequently analyzed specimens followed by pus (22.5\%), blood (20.5\%) and sputum (10.5\%). Other specimens analyzed were body fluids $(5.2 \%)$, stool $(0.4 \%)$ and ET Tip and secretion $(0.3 \%)$. Presence of high number of urine sample indicated the high urinary tract infection among the patient visiting the hospital. Similar pattern of specimen distribution was observed in other similar studies (Bhandari 2011; Upadhyaya 2015 ; Ghimire 2016). In almost all hospital based studies urine was found to be the most frequently analysed specimen (Bomjan 2005; Poudyal 2010; Gautam 2015; Ghimire 2016).

Of the total 200 isolates of Enterobacteriaceae, E. 
coli was the the predominant pathogen with 56.5\% which was comparable with the result of Mulla (2012) i.e, 55.6\%. Ghimire (2016) reported 53.8\% but high prevalence i.e, $73.4 \%$ of E. coli reported by Panta (2012) in the similar study. Following E. coli, K. pneumoniae was found to be the most frequently isolated bacteria with $31.5 \%$ which was comparable with the result of Mulla (2012) i.e, 31.2\%. Ghimire (2016) reported 34.6\% but very low prevalence of K. pneumoniae was reported by Panta (2012) in the similar study. In comparison of $4 \%$ of Proteus species isolated by Mangaiarkkarasi et al. (2013), 5.5\% of P. mirabilis and 3.5\% P. vulgaris were isolated in our study. But higher prevalence of Enterobacter (7.9\%) was found in the study of Mulla (2012) in comparison to our study (1.5\%). Other bacteria isolated were S. Paratyphi, Citrobacter spp. and Serratia spp. $0.5 \%$ each. Sample from patients under probable treatment, sample transport delay, sampling errors or nosocomial transmission could be the possible reasons for some variable results in our study

In this study all the isolated strains of Enterobacteriaceae isolates were tested with specific antibiotics according to CLSI 2014. Among the 12 different antibiotics used against all the isolates of Enterobacteriaceae , imipenem (89.5\%) was found to be the most effective antibiotic followed by amikacin (73\%) which was in accordance with the similar study conducted by Ghimire (2016). Nitrofurantoin was found to be $77.9 \%$ sensitive among urinary Enterobacteriaceae isolates. Similarly in a study antibiotic sensitivity pattern of the isolates revealed that $89.5 \%$ of the isolates were sensitive to imipenem, $73 \%$ were sensitive to amikacin followed by chloramphenicol (69.5\%), and tetracycline $(52.5 \%)$ whereas high resistance was seen for ampicillin (98.5\%), cefixime (80.5\%), amoxyclav (77.5\%), ceftriaxone $(73.55 \%)$, cefotaxime $(72 \%)$, cefoxitin $(65 \%)$, cotrimoxazole (58\%) and ciprofloxacin (58\%) (Metri et al. 2011).

In this study a total of 200 isolates of Enterobacteriaceae were isolated from 1602 different clinical specimens processed. Thus, the prevalence of Enterobacteriaceae in various clinical specimens was found to be $12.5 \%$. Of the total isolates of Enterobacteriaceae, 171 (85.5\%) were found to be multidrug resistant. Similar study conducted in Sahid Gangalal Memorial Hospital by Ghimire (2016) showed $11.8 \%$ growth and $82.6 \%$ of them were MDR. In a study by Yadav (2015) showed high growth positivity of $31.3 \%$ and $96.8 \%$ multidrug resistance among them. Another study conducted by Panta (2012) at Tertiary Hospital showed 13.9\% growth positivity of Enterobacteriaceae with 59.7\% MDR among them. However, in a study by Baral (2008) rather high growth positivity of $22.4 \%$ was observed but the MDR among the isolates was only $40.7 \%$.

High drug resistance in Enterobacteriaceae is attributed to mutations in chromosomal genes ability to share genetic material and mobile resistant genes. The mobile genetic elements are responsible for capturing resistant genes from the chromosomes of a variety of bacterial species and then DNA molecules horizontally and vertically (Patridge 2015).

The high level of drug resistance seen among E. coli is mediated by beta-lactamases, which hydrolyzes the beta-lactam ring inactivating the antibiotic. The classical TEM-1, TEM-2 and SHV-1 enzymes are the predominant plasmid-mediated beta-lactames of Gram negative rods (Livermore 1995). Mutations at the target site i.e, gyrA, which is a gyrase subunit gene, and parC, which encodes a topoisomerase subunit, confer resistance to fluoroquinolones (Ozeki et al. 1997). In addition to this mechanism, there are more than efflux systems in E. coli that can export structurally unrelated antibiotics; these multidrug resistance efflux pump (MDR pump) systems contribute to intrinsic resistance for toxic compounds such as antibiotics, antiseptics, detergents and dyes (Sulavik et al. 2001).

Higher level of drug resistance seen in K. pneumoniae is mediated by the production of different kind of beta-lactamases primarily ESBL, AmpC and Metallo beta-lactamases. The fact that the carriage of resistance trait for quinolones and aminoglycoside in the plasmid along with the gene for beta-lactamases have had a great impact on the drug resistance character shown by this pathogenic bacteria (Thomson 2001; Walsh et al. 2005; Picao et al. 2008). The $a c r R$ and $\operatorname{ram} A$ genes are involved in expression of the MDR phenotype in strains of K. pneumoniae (Paudel 2013).

There are many mechanisms whereby Proteus spp. confer resistance to the drugs including impermeability and acquired resistance ass plasmids, transposons and mutations (Paudel 2013). However, the production of plasmid or chromosomal encoded beta-lactamase enzyme is the most common mechanism of resistance in Gram negative bacteria causing clinical significant infection (Bush et al. 1995). 
Of the total MDR Enterobacteriaceae isolates tested for screening of ESBL production, 132 (77.2\%) isolates were screened as positive. Of the total 132 screened positive isolates, 47 (33.8\%) isolates confirmed as ESBL producers. $24.1 \%(7 / 29)$ of Enterobacteriaceae isolates were NON-MDR but ESBL producers. The prevalence of ESBL producers among total MDR isolates was $27.5 \%$ (47/171). In similar studies Balan (2013), Batchoun et al. (2009) and Thenmozhi and Sureshkumar (2013) reported 23\%, 22.9\% and $17.7 \%$ ESBL producer respectively among total Gram negatve bacterial isolates. Among the total 54 ESBL positive isolates, majority of them were E. coli with $58.9 \%$ followed by K. pneumoniae 40.8\%, P. mirabilis $5.6 \%$ and Salmonella Paratyphi $1.9 \%$. In a similar study Khanal et al. (2013) ESBL detection was reported highest in E. coli.

Only $38.8 \%$ of the total 139 screen positive isolates for ESBL production were ESBL producer. The positive ESBL screening result may be due to AmpC betalactamases more often than to ESBL. It is difficult to detect ESBL in those isolates that typically have inducible AmpC chromosomal enzyme which may be induced by clavulanate and attack the indicator cephalosporin, thus masking any synergy arising from ESBL production. In case of Citrobacter spp, Serratia spp. Enterobacter spp. resistance to oxyimino-cephalosporin is due to mutational hyper production of chromosomal AmpC beta-lactamase production rather than ESBL (Beceiro et al. 2008; Livermore and Brown 2001).

\section{ACKNOWLEDGEMENTS}

Authors are thankful to Central Department of Microbiology, T.U., Kathmandu and National Medical College and Teaching Hospital, Birgunj.

\section{REFERENCES}

Balan K (2013) Detection of extended spectrum betalactamase among Gram negative clinical isolates from a tertiary care hospital in South India. Int $J$ Med Sci 1: 28-30.

Baral P, Neupane S, Marasini BP, Ghimire KR, Lekhak B and Shrestha B (2012) High prevalence of multidrug resistance in bacterial uropathogens from Kathmandu, Nepal. BMC Research Notes 5: 38.

Batchoun RG, Sweden SF and Shurman AM (2009) Extended spectrum beta-lactamases among Gram negative bacterial isolates from clinical specimens in three major hospitals in Northern Jordon Int J
Microbial 9: 513874.

Beceiro A, Fernandez-Cuenca F, Ribera A, MartinezMartinez L, Pascaul A, Vila J, Rodriguez-Bano J, Cisneros JM, Pachon J and Bou G (2008) False extended spectrum beta-lactamase detection in Acinetobacter spp due to intrinsic susceptibility to clavulanic acid. J Antimicrobial Chemoether 61: 301-308.

Bush K, Jacoby GA and Medeiros AA (1995) A functional classification scheme for beta-lactamases and its correlation with molecular structure. Antimicrob Agents Chemoether 39: 1211-1233.

Byarugaba DK (2004) A view on antimicrobial resistance in developing countries and responsible risk factors. Int J Antimicrob Agents24: 105-110.

Canton R and Coque TM (2006) The CTX-M betalactamases. Curr Opin Microbial 9: 466-475.

Fang D, Ping LU and Sangjie YU (2008) Characterization of multidrug-resistant and metallo $\beta$-lactamase producing Pseudomonas aeruginosa isolates from a paediatric clinic in China. Chinese Medical Journal 21: 1611-1616.

Gautam S (2015) Detection of biofilm formation and metallo beta-lactamases in P. aeruginosa. M.Sc. Dissertation Submitted to Central Department of Microbiology. Tribhuvan University. pp 36-44.

Ghimire A (2016) Extended spectrum beta-lactamase (ESBL) producing multidrug resistant Gram negative bacteria from various clinical specimens of patients visiting in a tertiary hospital. M.Sc. Dissertation Submitted to Central Department of Microbiology. Tribhuvan University. pp 24-43.

Gray LD (1995) Escherichia, Salmonella, Shigella and Yersinia, Manual of clinical microbiology. Murray PR, Baron EJ, Pfaller MA, Tenovar FC, Yolken RH (eds). Washington DC. pp 450-456.

Khan F, Khan A and Kazmi SU (2014) Prevalence of susceptibility pattern of multi drug resistant clinical isolates of Pseudomonas aeruginosa in Karachi. Pakistan Journal of Medical Science 30: 951954.

Livermore DM and Brown DFJ (2001) Detection of beta-lactamase mediated resistance. J Antimicrob Chemoether 48: 59-64. 
Livermore DM (2003) Bacterial resistance: origins, epidemiology and impact. Clin Infect Dis 36: 511523.

Metri BC, Jyothi P and Peerapur BV (2011) The prevalence of ESBL among Enterobacteriaceae in a tertiary care hospital of North Karnataka, India. J Clin Diagn Res 5: 470-475.

Ozeki S, Deguchi T, Yasuda M, Nakano M, Kawamura T, Nishino Y and Kawada Y (1997) Development of a rapid assay for detecting gyr $A$ mutations in E. coli and determination of incidence of gyr A mutations in clinical strains isolated from patients with complicated urinary tract infections. J Clin Microbial 35: 2315-2319.

Panta K, Ghimire P, Rai SK, Mukhiya RK, Singh RM and Rai G (2013) Antibiogram typing of Gram negative isolates in different clinical samples of a tertiary hospital. Asian Journal of Pharmaceutical and Clinical Research 6: 153-156.

Patridge SR (2015) Resistance mechanisms in Enterobacteriaceae pathology. J RCPA 47: 276-284.

Paudel S (2013) Status of extended spectrum betalactamase producing Enterobacteriaceae among uropathogens. M.Sc. Dissertation Submitted to Central Department of Microbiology. Tribhuvan University.pp 27-36.

Picao RC, Andrade SS, Nicoletti AG, Campana EH, Moraes GC, Mendes RE and Gales AC (2008) Metallo beta-lactamase detection: Comparative evaluation of double disk synergy versus combined disk tests for IMP-, GIM-, SIM-, SPMor VIM - producing isolates. J Inst Med 28: 19-27.

Poudyal S (2010) Prevalence of beta-lactamase producing multidrug resistant bacterial pathogens isolated from different clinical samples at National Public Health Laboratory. M. Sc. Dissertation Submitted to Central Department of Microbiology. Tribhuvan University. pp 44-58.

Rupp ME and Fey PD (2003) Extended spectrum $\quad \beta$-lactamases (ESBLs)-Producing Enterobacteriaceae. considerations for diagnosis, prevention and drug treatment, Drugs 63: 353365.

Sanders CC and Sanders WE (1987) Clinical importance of inducible beta-lactamases in Gram-negative bacteria. Eur J Clin Microbial 6: 435-438.

Thenmozhi S and Sureshkumar BT (2013) Prevalence of extended spectrum beta-lactamase producing Gram negative bacteria in private hospital, Tiruchengode, India. Int J Curr Microbial App Sci 2: $280-289$.

Thomson KS (2001) Controversies about ExtendedSpectrum and AmpC Beta-Lactamases. Emerg Infect Dis 7: 333-336.

Sulavik MC, Houseweart C, Cramer C, Jiwani N, Murgolo N, Greene J, Di Domenico B and Shimer G (2001) Antibiotic susceptibility profiles of $E$. coli strains lacking multidrug efflux pump genes. Antimicrob Agents Chemoether 45: 1126-1136.

Upadhyaya U (2015) Detection of beta-lactamase producing Gram negative bacteria in different clinical specimens of patients visiting tertiary level heart centre. M.Sc Dissertation Submitted to Central Department of Microbiology, Tribhuvan University. pp 28-36.

Walsh TR, Toleman MA, Poirel L and Nordmann P (2005) Metallo beta-lactamases, the quiet before the storm? Clin Microbial Rev 18: 306-325. 